\title{
Comparative study of silicone and polyurethane nephrostomy catheters used for long-term urinary drainage in malignancy
}

\author{
Nicolae GRIGORE, Valentin PIRVUT, Ionela MIHAI, Adrian HASEGAN \\ Department of Urology, Faculty of Medicine, "Lucian Blaga” University, Sibiu, Romania
}

\begin{abstract}
Despite the modern advancement in endourology, percutaneous nephrostomy play an important role in many urologic conditions, which of the most important is the relief of upper urinary tract in patients with malignancies that interests the ureter or the uretero-vesical junction.

The aim of the study is to compare the complications of polyurethane and silicone catheters used for percutaneous nephrostomy in patients with unilateral or bilateral uretero-hydronephrosis secondary to locally advanced abdomino-pelvin malignancies.

We have retrospective analyzed 164 patients (p), admitted between January 2013 and December 2016 in Urology Department Sibiu, who benefit from unilateral or bilateral percutaneous nephrostomy for secondary uretero-hydronephrosis with acute renal failure.
\end{abstract}

Keywords: percutaneous nephrostomy, catheter, polyurethane, silicone, uretero-hydronephrosis

\section{INTRODUCTION}

Despite the modern advancement in endourology percutaneous nephrostomy play an important role in many urologic conditions, which of the most important is the relief of upper urinary tract in patients with malignancies that interests the ureter or the uretero-vesical junction.

Malignant urinary obstruction can be secondary to extrinsic tumor compression, direct tumor invasion or an intrinsic genitourinary (GU) malignancy. One large study reported malignant obstruction as the indication for over $60 \%$ of all nephrostomies (1).

Percutaneous nephrostomy is a gentle procedure associated with high technical success and low morbidity. The complications of percutaneous nephrostomy consists in urinary tract infection, catheter dislodgement, catheter ob- struction by debris, urinary leakage and inflammation of the skin at the site of insertion of the percutaneous catheter (2).

\section{MATERIAL AND METHODS}

In the present study we analyzed in a comparative manner the complications of polyurethane and silicone catheters used for percutaneous nephrostomy in patients with unilateral or bilateral uretero-hydronephrosis secondary to locally advanced abdominopelvic malignancies.

We have retrospective analyzed 164 patients (p), admitted between January 2013 and December 2016 in Urology Department Sibiu, who benefit from unilateral or bilateral percutaneous nephrostomy for secondary uretero-hydronephrosis with acute renal failure. We used for 
nephrostomy two types of catheters: 1 . A polyurethane Cope loop $12 \mathrm{Fr}$ or $14 \mathrm{Fr}$ catheter; 2. 2 way silicone Foley $12 \mathrm{Fr}$ or $14 \mathrm{Fr}$ catheter. The placement of the catheters was done using a combined ultrasound and fluoroscopic technique. Intraoperative urine cultures were collected using the puncture needle. The catheters were changed every 3 months or earlier if needed using the fluoroscopic guidance. We evaluated the patients at every catheter change over an exact period of time of 1 year. At every evaluation urine culture were collected 1-2 hours before removing the nephrostomy catheters, and after removal all catheters were microscopically analyzed for encrustation and using a hydrophilic guidewire for patency. Patients who could not complete the follow-up at 1 year were excluded from the present study.

The statistical analysis of this paper was done by using chi-square test, the Fischer exact test. A p value $<0.05$ was considered statistically significant.

\section{RESULTS}

The mean age of patients $(p)$ was 67.8 years (range $39-91$ years) and $65.2 \%$ (107/164) were women. Bilateral percutaneous nephrostomy was performed in $75.6 \%$ cases (124/164). Polyurethane nephrostomy catheters were used in $54.2 \%$ p (89/164). The characteristics are presented in Table 1.

When comparing the need of catheter replacement before pre-established visit, $17.1 \%$ $\mathrm{p}$ required at least one catheter change, with higher rate in the silicone group (Table 2).

The most common reason of catheter replacement was the obstruction due to debris with a rate of $4.9 \%(60 / 1222)$, followed by catheter dislodgement with a rate of $1.1 \%$ (11/1222).

The obstructed catheter rate was higher in the silicone catheter group with a rate of $7.5 \%$ (9.7\% for $12 \mathrm{Fr}$ and $4.8 \%$ for $14 \mathrm{Fr}$ ) compared to polyurethane catheters that had a rate of $2.4 \%$ (2.7\% for $12 \mathrm{Fr}$ and $2 \%$ for $14 \mathrm{Fr}$ ) (Table 3 ).

TABLE 1

\begin{tabular}{|c|c|c|c|c|}
\hline & All patients & Polyurethane chatheter & Silicone catheter & p \\
\hline Patients & 164 & 89 & 75 & \\
\hline Mean age $(y r) \pm S D$ (range) & $67.8 \pm 19.4(39-91)$ & $66.7 \pm 17.6(39-88)$ & $68.1 \pm 16.4(42-91)$ & ns \\
\hline \multicolumn{5}{|l|}{ Sex } \\
\hline Women & $107(65.2 \%)$ & $56(62.9 \%)$ & $51(68 \%)$ & ns \\
\hline Men & $57(34.8 \%)$ & $33(37.1 \%)$ & $24(32 \%)$ & ns \\
\hline \multicolumn{5}{|l|}{ Catheter placement } \\
\hline Unilateral & $40(24.4 \%)$ & $22(24.8 \%)$ & $18(24 \%)$ & ns \\
\hline Bilateral & $124(75.6 \%)$ & $67(75.2 \%)$ & $57(76 \%)$ & ns \\
\hline \multicolumn{5}{|l|}{ Catheter diameter } \\
\hline \begin{tabular}{l|l}
$12 \mathrm{Fr}$ \\
\end{tabular} & $111(67.6 \%)$ & $71(79.7 \%)$ & $40(53.3 \%)$ & ns \\
\hline $14 \mathrm{Fr}$ & $53(32.4 \%)$ & $18(20.3 \%)$ & $35(46.7 \%)$ & ns \\
\hline Intraoperative positive culture & $18(10.9 \%)$ & $8(8.9 \%)$ & $10(13.3 \%)$ & ns \\
\hline
\end{tabular}

ns - not statistically significant

TABLE 2

\begin{tabular}{|l|c|c|c|c|c|c|}
\hline \multirow{2}{*}{} & \multicolumn{2}{|c|}{ Polyurethane chatheter (p) } & \multicolumn{2}{|c|}{ Silicone catheter (p) } & \multirow{2}{*}{ All patients } & \multirow{2}{*}{$\mathbf{p}$} \\
\cline { 2 - 5 } & $\mathbf{1 2} \mathbf{~ F r}$ & $\mathbf{1 4} \mathbf{~ F r}$ & 12 Fr & 14 Fr & & ns \\
\hline $\mathrm{V}=4$ & $66(92.9 \%)$ & $15(83.3 \%)$ & $27(67.5 \%)$ & $28(80 \%)$ & $136(82.9 \%)$ & $\mathrm{ns}$ \\
\hline $\mathrm{V} \mathrm{5-7}$ & $4(5.7 \%)$ & $3(16.7 \%)$ & $9(22.5 \%)$ & $5(14.3 \%)$ & $21(12.8 \%)$ & $\mathrm{ns}$ \\
\hline $\mathrm{V} \geq 8$ & $1(1.4 \%)$ & 0 & $4(10 \%)$ & $2(5.7 \%)$ & $7(4.3 \%)$ & $\mathrm{ns}$ \\
\hline Total & 71 & 18 & 40 & 35 & 164 & $\mathrm{~ns}$ \\
\hline
\end{tabular}

ns - not clinically significant, $\mathrm{V}-$ visit

TABLE 3

\begin{tabular}{|l|c|c|c|c|c|c|}
\hline \multirow{2}{*}{} & \multicolumn{2}{|l|}{ Polyurethane chatheter (n) } & Silicone catheter (n) & \multirow{2}{*}{ Catheters placed } & \multirow{2}{*}{ p } \\
\cline { 2 - 6 } & $\mathbf{1 2} \mathbf{~ F r}$ & $\mathbf{1 4} \mathbf{~ F r}$ & $\mathbf{1 2 ~ F r}$ & $\mathbf{1 4 ~ F r}$ & & \\
\hline Catheters used & 441 & 200 & 318 & 267 & 1222 & \\
\hline Obstructed catheter (\%) & $12(2.7 \%)$ & $4(2 \%)$ & $31(9.7 \%)$ & $13(4.8 \%)$ & $60(4.9 \%)$ & 0.031 \\
\hline Catheter dislodgement & $1(0.2 \%)$ & 0 & $7(2.2 \%)$ & $6(2.2 \%)$ & $14(1.1 \%)$ & 0.022 \\
\hline Total & \multicolumn{2}{|c|}{$17(2.6 \%)$} & \multicolumn{2}{|c|}{$57(9.9 \%)$} & $74(6 \%)$ & \\
\hline
\end{tabular}

ns - not clinically significant 


\begin{tabular}{|c|c|c|c|c|c|c|}
\hline & \multicolumn{2}{|c|}{ Polyurethane chatheter (n) } & \multicolumn{2}{|c|}{ Silicone catheter (n) } & \multirow{2}{*}{$\begin{array}{l}\text { Catheters } \\
\text { placed }\end{array}$} & \multirow{2}{*}{$\mathbf{p}$} \\
\hline & $12 \mathrm{Fr}$ & $14 \mathrm{Fr}$ & $12 \mathrm{Fr}$ & $14 \mathrm{Fr}$ & & \\
\hline Standard catheters used & 441 & 200 & 318 & 267 & 1222 & \\
\hline Urinary infection & $83(18.8 \%)$ & $33(16.5 \%)$ & $51(16 \%)$ & $49(18.3 \%)$ & $216(17.6 \%)$ & 0.8 \\
\hline Catheter encrustation & $126(28.5 \%)$ & $59(29.5 \%)$ & $98(30.8 \%)$ & $74(27.7 \%)$ & $357(29.2 \%)$ & 0.7 \\
\hline Skin inflammation & $48(10.8 \%)$ & $18(9 \%)$ & $9(2.8 \%)$ & $11(4.1 \%)$ & $86(7 \%)$ & 0.041 \\
\hline
\end{tabular}

The catheter dislodgement in our study group was specific to the silicone catheter group with a rate of $2.2 \%$, with only one case of dislodgement in the polyurethane group (0.2\%).

The complications of nephrostomy placement included urinary infection (17.6\%), catheter encrustation (29.2\%) and local skin inflammation at the site of nephrostomy (7\%). (Table 4).

\section{DISCUSSION}

Improvement of renal function after PCN can be of clinical benefit in patients who might be cured or for prolonged palliative care (4).

The ideal nephrostomy tube is yet to be discovered, in meantime the type of nephrostomy to be used the urologist choice depending on the biomaterial used and by personal experience.

We did not experience malposition of nephrostomy because we used a combined ultrasound and fluoroscopic technique to place the nephrostomy tube.

Overall, 18 patients of 164 (10.9\%) presented positive urine culture at the moment of nephrostomy.

The silicone nephrostomy tube are more predisposed for replacement $(9.9 \%)$ than the polyurethane catheter (2.6\%), due to debris obstruction and catheter dislodgement.

When talking about the obstructed catheter the result can be explained by the fact that the lumen of a $12 \mathrm{Fr}$ silicone catheter is smaller than a $12 \mathrm{Fr}$ polyurethane catheter, same for the 14 Fr catheter (Fig. 1).

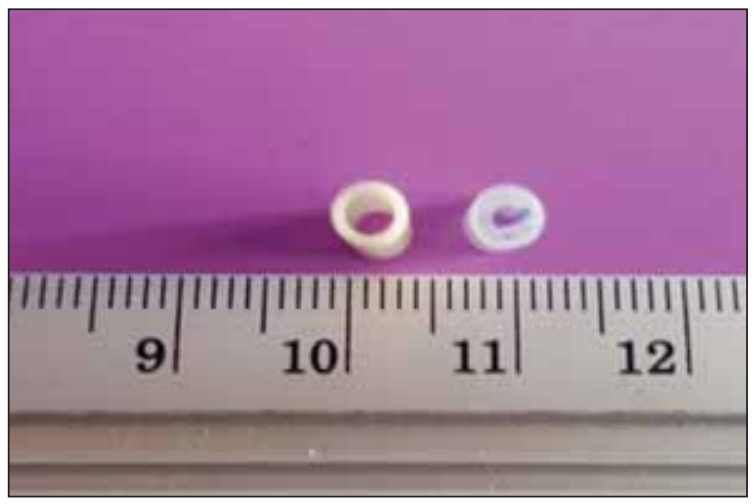

FIGURE 1. Polyurethane catheter (left) and silicone catheter (right) crossing section
Bayne et al. reported that the type of the catheter do not influence the dislodgement rate, but that was a study on short postoperative time catheter placement (3). But this study was done for patient that needed a short time nephrostomy. In our series the rate of dislodgement is higher in the silicone catheters $(2.2 \%$ vs. $0.2 \%$ ). We put this result on the fact that we used 2 way silicone catheters with $5 \mathrm{ml}$ intrarenal balloon that was predisposed for deterioration and rupture, that being the reason of dislodgement. The polyurethane catheters were fixed directly to the skin, which seems to be safer than the intrarenal balloon system.

Another complication related to the biomaterial used in the manufacturing of the nephrostomy is the skin inflammation at the site of the insertion of the catheter, which was more present in patients with polyurethane type (10.2\%) than in silicone group (3.4\%).

Complications of PCN insertion occur in $4-26 \%$ of procedures (5-8) and include malposition, dislodgement or occlusion of the tube. Occlusion may result from blood clot formation in the renal pelvis. In our series if we take into account only the dislodgement and catheter occlusion the complication rate is $6 \%$, but if we add the local skin inflammation to the complications the rate will rise to $11 \%$.

\section{CONCLUSIONS}

Percutaneous nephrostomy is a safe, minim invasive, cheap and quick method of urinary diversion in patients with acute renal failure due to ureteral obstruction because of malignant disease of pelvic origin.

Our data suggest that for long term nephrostomy polyurethane catheters represents a better alternative for patients than silicone catheters, with lower rate of dislodgement and occlusion.

Local skin inflammation, catheter encrustation, better modalities of fixation and urinary infection due to catheter placement are important factors to take into account for developing a new generation of nephrostomy catheters. 
Acknowledgements: The authors declare no conflict of interests and no sponsorship was provided by the manufacturer of the catheters in- volved in this study. All authors have read and approved this publication and had equal scientific contribution in publishing this material.

\section{REFERENCES}

1. Farrell T.A., Hicks M.E. A review of radiologically guided percutaneous nephrostomies in 303 patients. J Vasc Interv Radiol. 1997; 8(5):769-774.

2. Radecka E., Magnusson A., Acta Radiol. 2004 Apr; 45(2):184-8.

Complications associated with percutaneous nephrostomies. A retrospective study.

3. Bayne D., Taylor E.R., Stoller M.L. et al. Determinants of Nephrostomy Tube Dislodgment After Percutaneous Nephrolithotomy. J Endourol. 2015 Mar; 29(3):289-292.
4. Plesinac-Karapandzic V., Masulovic D., Markovic B., Djuric-Stefanovic A., Plesinac S., Vucicevic D., Milovanovic Z., Milosevic Z. Percutaneous nephrostomy in the management of advanced and terminal-stage gynecologic malignancies: outcome and complications. Eur J Gynaecol Oncol. 2010; 31(6):645-50.

5. Dowling R.A., Carrasco C.H., Babaian R.J. Percutaneous urinary diversion in patients with hormone-refractory prostate cancer. Urology 1991; 37:89-91

6. Markowitz D.M., Wong K.T., Laffey K.J., Bixon R., Nagler H.M., Martin E.C.
Maintaining quality of life after palliative diversion for malignant ureteral obstruction. Urol Radiol 1989; 11:129-32

7. Lau M.W., Temperley D.E., Mehta S., Johnson R.J., Barnard R.J., Clarke N.W. Urinary tract obstruction and nephrostomy drainage in pelvic malignant disease. Urology 1995; 76:565-9

8. Naricula J., Murphy D.G., Jenner C., Sellars N., Gwyther S., Gordon S.G., Swinn M.J. Nephrostomy insertion for patients with bilateral ureteric obstruction caused by prostate cancer. The British Journal of Radiology, July 2009; 571-576 